\title{
Drug Thrombus Resulting in Superior Vena Cava Syndrome: A Case Report
}

\author{
Stijn Vanstraelen ${ }^{1}$, Jeroen Vandenbrande ${ }^{2}$, Alaaddin Yilmaz ${ }^{2}$ \\ 1 Katholieke Universiteit Leuven Universitaire Ziekenhuizen Leuven, Leuven, Belgium \\ 2 Jessa Hospital, Hasselt, Belgium
}

\begin{abstract}
Introduction: Superior vena cava syndrome is one of the more serious complications of central venous catheter insertion. Drug interactions of administered drugs used in association with these catheters can lead to formation of precipitations and consequently thrombus formation. These interactions can be either anion-cation or acid-base based and more commonly present in clinical practice than expected. Case presentation: The case of a 31-year old female who was admitted to an intensive care unit with an intracranial haemorrhage, is presented. Occlusion of the superior vena cava was caused by a drug-induced thrombus, formed by the precipitation and clotting of total parenteral nutrition and intravenous drugs. Given the nature of the thrombus and a recent intracranial haemorrhage, the patient was treated with a central thrombectomy supported by a heparin-free extracorporeal membrane oxygenation. Conclusion: Knowledge of drug interactions is crucial in order to heighten awareness for the dangers of concomitant drug administration, especially in combination with total parenteral nutrition in critically ill patients.
\end{abstract}

Keywords: upper extremity deep vein thrombosis, superior vena cava syndrome, central venous catheter

Received: 2 January 2020 / Accepted: 20 March 2020

\section{INTRODUCTION}

Central venous catheter (CVC) placement is increasingly routinely performed in-intensive care units (ICU), as well as outside ICUs, since the need for chemotherapy, total parenteral nutrition (TPN) and concomitant or long-term drug therapy has increased [1].

An estimated $18 \%$ of patients in ICUs, receiving a subclavian CVC, experience adverse mechanical events, such as hematoma, pneumothorax and arterial puncture [2]. Superior vena cava (SVC) thrombosis is reported in 1.5 to $21 \%$ of patients in ICUs; however, this usually remains asymptomatic and only detected on venography or ultrasonography $[2,3,4]$.

A rare case of SVC syndrome, caused by a drug thrombus which required surgical thrombectomy is reported. A drug thrombus is defined as a thrombus predominantly consisting of an aggregation of chemicals or drugs. These aggregates are usually formed of chemical acid-base and anion-cation reactions $[5,6]$.

\section{DESCRIPTION OF THE CASE}

A 31year-old female of $63 \mathrm{~kg}$ was admitted to the regional general hospital, Hasselt, Belgium. On admis- sion, no significant previous medical history was recorded; an intracranial haemorrhage in the left temporal lobe after a low velocity fall was suspected and confirmed by computed tomography (CT) of the brain. Her Glasgow coma scale was 3/15. She was intubated and an external ventricular drain was inserted in the operating theatre the same day.

On admission to the ICU, continuous infusions of Diprivan $^{\odot}$ (Astra Zeneca, Macclesfield, United Kingdom) $(1 \%, 6 \mathrm{mg} / \mathrm{kg} / \mathrm{h}$, intravenous infusion) and Dipidolor $^{\odot}$ (Johnson\&Johnson, New Brunswick, United States) $(0.1 \mathrm{mg} / \mathrm{kg}$, intravenous infusion) were started for sedation and analgesia purposes, as well as Pentothal $^{\odot}$ (Abbott, Chicago, United States) $(1.5 \mathrm{mg} / \mathrm{kg}$, intravenous infusion) and Dormicum ${ }^{\odot}$ (Cheplapharm Arzneimittel, Mesekenhagen, Germany) $(0.1 \mathrm{mg} / \mathrm{kg} / \mathrm{h}$, intravenous infusion) to control intracranial pressure and prevent epilepsy. Additionally, she received Pantomed $^{\odot}$ (Takeda Tokyo, Japan) (40mg, intravenous infusion during $15-30 \mathrm{~min}$ ) once daily as a prevention for gastric ulcers. These were continued throughout the whole hospitalization.

Due to gradually increasing intracranial pressure, a decompressive craniotomy was performed the day af- 
ter admission. Intravenous Augmentin ${ }^{\odot}$ (GlaxoSmithKline, Brentford, United Kingdom) (1g, intravenous infusion every $6 \mathrm{~h}$ ) was started on day two after admission, as targeted therapy for a ventilator-associated pneumonia. Sputum Cultures confirmed an omnisensitive Staphylococcus Aureus and antibiotic therapy was continued for ten days.

Due to refractory gastroparesis, continuous total parenteral nutrition (Olimel N7E ${ }^{\odot}$, Baxter, Deerfield, United States) (1.5L, $60 \mathrm{ml} / \mathrm{h}$, intravenous infusion) was administered as of day three post admission,

During her admission she intermittently received Nimbex ${ }^{\odot}$ (Abbvie, Chicago, United States) $(0.15 \mathrm{mg} / \mathrm{kg}$, continuous intravenous infusion) for two days postoperatively while on ventilator support. Furthermore, Solu-Cortef ${ }^{\odot}$ (Pfizer, New York City, United States) (100mg, intravenous during 15-30min, once daily) and dexamethasone (Mylan, Canonsburg, United States) (4mg, intramuscular, every $6 \mathrm{~h}$ ) were administered from day one until day fourteen post-admission, to decrease cerebral oedema. Finally, mannitol (Pfizer, New York City, United States) $(0.2 \mathrm{~g} / \mathrm{kg}$, intravenous infusion during $30 \mathrm{~min}$, once daily) and Lasix ${ }^{\odot}$ (Sanofi, Paris, France) (40mg, intravenous infusion during 15$30 \mathrm{~min}$, once daily) were administered from day three to five post-admission, and on day thirteen to day fifteen post-admission to stimulate diuresis and achieve euvolemia postoperatively.
On day ten, post-admission, due to an elevated temperature of $39.3^{\circ} \mathrm{C}$, the $\mathrm{CVC}$ was removed and replaced with a triple lumen CVC in the right subclavian vein under ultrasound guidance with the tip at de SVC/right atrial junction [7].

De-escalation of infusion rate was noted overnight with only two lumens available for drug administration. The following day, severe oedema of the upper extremities with a concomitant steep incline in intracranial pressure and compromised cerebral perfusion pressure was observed. At that time, a haemoglobin level of $12.4 \mathrm{~g} / \mathrm{dL}$, white blood cell count of $17.3^{\star} 10^{9} / \mathrm{L}$, platelet count of $185^{\star} 10^{9} / \mathrm{L}$, International Normalized Ratio of 1.2 and a fibrinogen level of $2.31 \mathrm{~d} / \mathrm{L}$, was recorded.

A bedside transoesophageal echocardiography (TEE) revealed a thrombus in the proximal SVC. (Figure 1)

CT confirmed a thrombus at the tip of the CVC, extending into the SVC, innominate and right subclavian veins.

The thrombus showed an irregular configuration and atypically high Hounsfield density.

A multidisciplinary decision was taken in the afternoon of day 11 post-admission to perform a surgical thrombectomy in order to relieve the SVC syndrome and prevent major lung embolus, since neither throm-

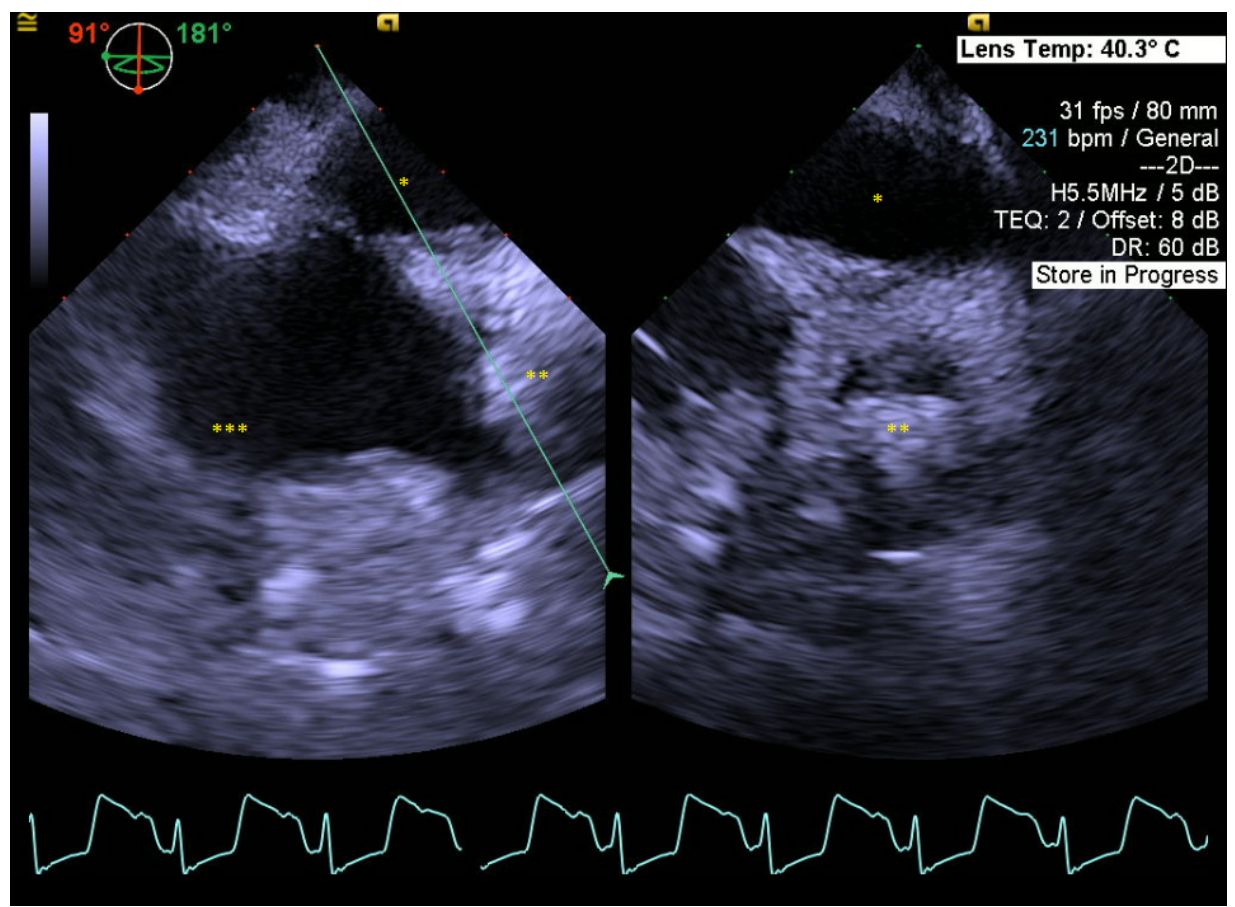

Fig. 1. Midesophageal bicaval view and midesophageal short-axis view of the SVC (obtained by explaining the bicaval view). * Right ventricle, ** Thrombus, *** Right atrium 
bolysis, nor trans catheter clot removal could be performed, due to the necessity of anticoagulation in a patient with a recent intracranial haemorrhage.

Following median sternotomy, the patient was started on extracorporeal membrane oxygenation (ECMO) (Medtronic, Minneapolis, MN, USA), to avoid heparinization, because of the intracranial haemorrhage, and provide hypothermic cardiac arrest. Subsequently, the right atrium was opened and a white/yellow grainy structure obstructing the lumen was identified instantly. (Figure 2).

Following thrombectomy, normal venous drainage ensued with immediate resolution of the SVC syndrome. A postsurgical TEE revealed no residual thrombus and a laminar flow to the right atrium.

The removed thrombus was divided into three pieces. The first sample was sent for culture at our own microbiology laboratory, which remained free from bacterial and fungal growth for four weeks postoperatively. The second sample was stained with haematoxylin and no cellular or organic material was detected. The third piece was sent to the pharmacological department of the associated University Hospital (UZ Leuven, Leuven, Belgium), because of a high suspicion of drug

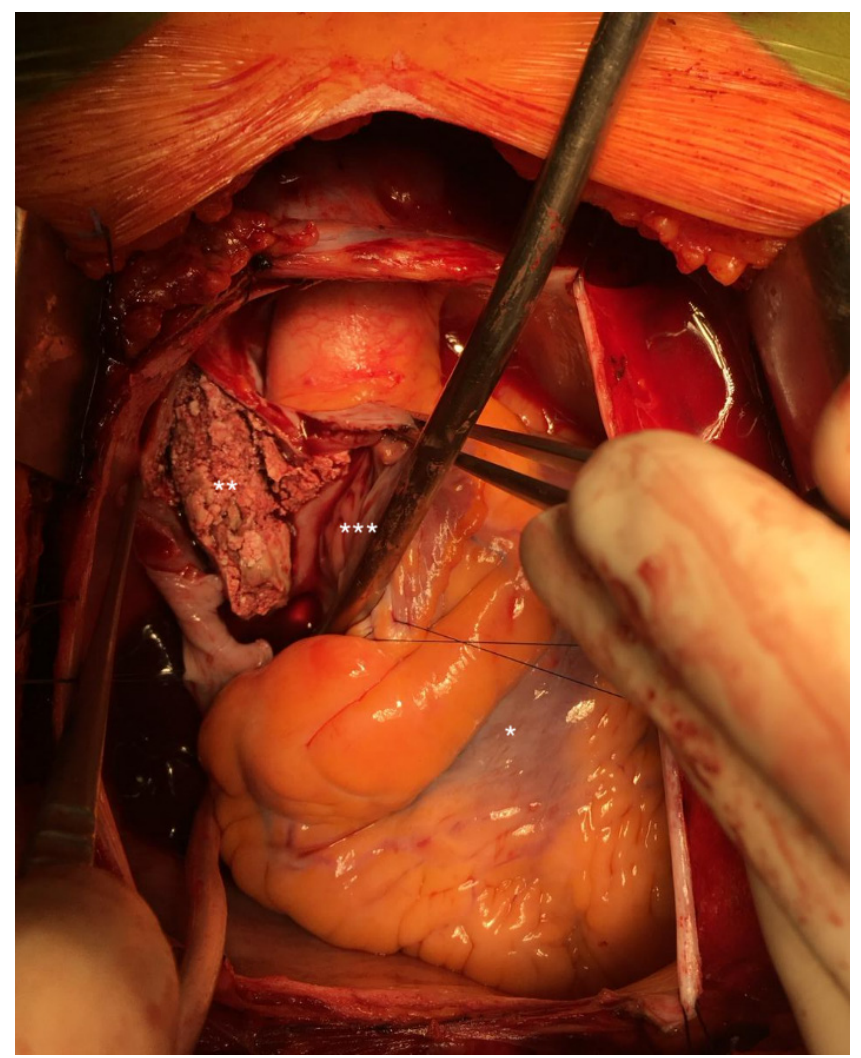

Fig 2. Operative image.

* Right ventricle, ${ }^{* *}$ Thrombus, ${ }^{* * *}$ Right atrium components in the thrombus. There, solubility, spectrophotometric and chromatographic analysis tests were undertaken. Eight weeks later, results of these final test showed a high phosphate concentration related to TPN and trapped active components of thiopental, pentobarbital, midazolam, laudanosine and piritramide in the aggregated specimen.

Despite the team's best effort, on the fourth postoperative week and 42 days after admission, the patient died from ischemia of the left cerebral cortex due to excessive intracranial pressure.

\section{DISCUSSION}

SVC thrombosis remains one of the more severe complications following the insertion of a CVC. The patient in this report had a rare type of thrombus, presumably formed out of aggregates of TPN and one or several other administered drugs. These aggregates can be the result of chemical reactions such as anion-cation and acid-base reactions. Weak bases and acids are known to form soluble salts in both low and high $\mathrm{pH}$ environments.

When mixed with a buffered substance, such as TPN, non-soluble precipitations can be produced. Most organic drugs are anions, which have the ability to interact with the hydronium ions found in TPN. Furthermore, bivalent anions are known to form poorly soluble salts with calcium $[5,6]$.

Therefore, numerous drugs have the potential to interact with each other, as well as components of TPN. Incompatibility reactions occur with midazolam, furosemide and cefazolin [8].

Midazolam as well as furosemide were administered to the current patient which had the potential to form a drug thrombus.

One type of precipitate occurs immediately, due to the poor mixing of the compounds; a second type within 24 to 48 hours due to crystal formation between calcium and bivalent bases [9].

Temperature also plays an important role in the precipitation process. Higher temperatures cause more free calcium as well as bibasic salt formation, both with the potential to create interactions $[5,9]$.

Our patient experienced elevated body temperatures, which could have accelerated the process of anion-cation formation. 
Ultimately, it was presumed that several factors contributed to the drug thrombus formation. First of all, the concomitant administration of TPN and incompatible drugs, combined with an increased availability of precipitating components due to the elevated body temperature. Furthermore, it was hypothesized that a snowball effect was created because of reduced flow over the catheter by the initial aggregates which subsequently prolonged contact time of the different elements. This could have led to the rapid evolution of a total occlusion. In this case, pantoprazole, midazolam, amoxicillin, mannitol and furosemide all had the potential to form precipitates with TPN separately or in combination.

TEE was the first diagnostic test used in the current case to detect the thrombus. This was later was confirmed via CT scan. Colour flow doppler ultrasound contributes to an increased detection rate of CVC related thrombi with a positive predictive value of $100 \%$ and a negative predictive value of $91 \%$ of total central vein occlusion $[3,7,10,11]$.

Diagnosis can be made indirectly by detection of thrombosis in the internal jugular vein or through flow voidance and nonpulsatile, non-phasic flow in the upper central veins [12-14]. Early detection is considered important because of the risk for pulmonary emboli when particles of the thrombus detach. Monreal et al. [15] found an incidence of pulmonary embolism related to CVC thrombi of $15 \%$, of which 1 in 3 were symptomatic

In retrospect, the unusual Hounsfield density and configuration of the thrombus on CT scan also suggested its atypical nature and contributed to the diagnosis.

Several treatment options exist as a treatment for SVC thrombus. Factor Xa inhibition is the first choice in stable patients with limited thrombus. Thrombolysis or interventional radiology guided clot retrieval can be considered in more unstable patients $[15,16]$.

Due to intracranial haemorrhage and necessity for anticoagulation, these options were not considered. Surgery frequently is a last resort when other treatment options are not feasible or fail [16].

Additionally, it has been shown that once precipitates are formed, they are unlikely to dissolve on their own [9], ultimately supporting our decision to proceed to surgery.
In the present case, a heparin-free ECMO circuit was used to provide hypothermic cardiac arrest and the necessary oxygenation to the brain. This method also minimized the need for heparinization as well as pathophysiologic stress. Muellenbach et al. [17] described a case series of heparin-free ECMO use in traumatic brain injury, with running times of up to 5 days without thromboembolic events.

On the other hand, cardiopulmonary bypass was not considered an option, as it would have required an activated clotting times of at least 400 seconds.

In conclusion, a unique case involving a rare type of thrombus resulting from the precipitation of concomitant drug therapy has been reported and described, which may contribute to heightened awareness with similar findings of SVC syndrome and thrombi resistant to anticoagulation.

\section{CONFLICT OF INTEREST}

None to declare.

\section{REFERENCES}

1. Ruesch S, Walder B, Tramer MR. Complications of central venous catheters: internal jugular versus subclavian access-a systematic review. Crit Care Med. 2002;30:454-60

2. Merrer J, De Jonghe B, Golliot F, et al. Complications of femoral and subclavian venous catheterization in critically ill patients: a randomized controlled trial. JAMA. 2001;286:700-7

3. Kuter DJ: Thrombotic complications of central venous catheters in cancer patients. Oncologist. 2004;9(2):207-16

4. McGee DC, Gould MK. Preventing complications of central venous catheterization. N Engl J Med. 2003;348:1123-33

5. Mühlebach S. Basics in clinical nutrition: Drugs and nutrional admixture. E Spen Eur E J Clin Nutr Metab. 2009;4:134-36.

6. Newton DW. Y-site Compatibility of Intravenous Drugs With Parenteral Nutrition. JPEN J Parenter Enteral Nutr. 2013;37(3): 287-99.

7. Pittiruti $M$, Hamilton $H$, Biffi $R$, MacFie J, Pertkiewicz $M$, ESPEN. ESPEN Guidelines on parenteral Nutrition: Central venous catheters (access, care, diagnosis and therapy of complications). Clin Nutr. 2009;28(4):365-77.

8. Trissel LA, Gilbert DL, Martinez JF, Baker MB, Walter WV, Mirtallo JM. Compatibility of parenteral nutrient solutions with selected drugs during simulated Y-site administration. Am J Health Syst Pharm. 1997;54(11):1295-300.

9. Allwood MC, Kearney MC. Compatibility and stability of additives in parenteral nutrition admixtures. Nutrition. 1998;14(9):697-706. 
Available online at: www.jccm.ro

10. Khouzam RN, Minderman D, D'Cruz IA. Echocardiography of the superior vena cava. Clin Cardiol. 2005;28(8):362-66.

11. Patel MC, Berman LH, Moss HA, McPherson SJ. Subclavian and internal jugular veins at Doppler US: abnormal cardiac pulsatility and respiratory phasicity as a predictor of complete central occlusion. Radiology. 1999;211:579-83.

12. Falk RL, Smith DF. Thrombosis of upper extremity thoracic inlet veins: diagnosis with duplex Doppler sonography. AJR Am J Roentgenol. 1987;149:677-82.

13. Wang S, Raio C, Nelson M. Bilateral internal jugular vein thrombosis. J. Clin Ultrasound. 2011;3(3):161-62.

14. Birch A, Um D, Laselle B. Ultrasound Detection of Superior
The Journal of Critical Care Medicine 2020;6(2) • 119 Vena Cava Thrombus. West J Emerg Med. 2014;15(6):715-18.

15. Monreal M, Raventos A, Lerma R, et al. Pulmonary embolism in patients with upper extremity DVT associated to venous central lines - a prospective study. Thromb Haemost. 1994;72: 548-50.

16. Burns KE, McLaren A. A critical review of thromboembolic complications associated with central venous catheters. Can J Anaesth. 2008;55(8):532-41.

17. Muellenbach RM, Kredel M, Kunze E, et al. Prolonged heparinfree extracorporeal membrane oxygenation in multiple injured acute respiratory distress syndrome patients with traumatic brain injury. J Trauma Acute Care Surg. 2012;72(5):1444-47. 